\title{
ESTIMATION DE LA PRECISION DES MOUVEMENTS \\ PROPRES DONNES DANS UN CATALOGUE D'ETOILES A \\ L'AIDE DES OBSERVATIONS VISUELLES DE COUPLES \\ STELLAIRES OPTIQUES
}

J. DOMMANGET

Observatoire Royal de Belgique, Belgium

\begin{abstract}
It is shown by means of several examples, that the visual observations of the relative rectilinear motion of components of wide optical double stars can be used to estimate the precision of catalogue proper motions.
\end{abstract}

Les nombreuses observations visuelles généralement disponibles pour tout couple stellaire dont la séparation atteint plusieurs dizaines de secondes d'arc (il s'agit alors généralement de couples optiques) permettent une bonne détermination du mouvement relatif de ses composantes. Le calcul, par la méthode des moindres carrés, de la trajectoire relative rectiligne, conduit à une précision généralement supérieure à celle des différences des mouvements propres donnés pour leurs composantes dans les catalogues stellaires de position, car les observations visuelles de ces couples sont non seulement assez nombreuses, mais couvrent souvent des intervalles de temps importants de l'ordre du siècle (figures la et $1 \mathrm{~b}$ ). Aussi, ces déterminations de trajectoires rectilignes devraient permettre de juger de la qualité des mouvements propres donnés dans les catalogues en comparant ceux-ci aux mouvements relatifs ainsi calculés.

Pour nous assurer des possibilités réelles de cette méthode, nous avons d'abord dressé la liste des couples pour lesquels une trajectoire précise avait été calculée (Dommanget et Nys, 1964) et pour lesquels aussi, les mouvements propres de chacune des composantes sont donnés dans trois catalogues: le 'Eigen-Bewegung Lexikon', le 'Catalogue de Yale' et le 'Catalogue du Smithsonian Astrophysical Observatory'.

Mais le matériel recueilli s'avérant par trop restreint (une dizaine de couples) contrairement à nos espérances - nous avons calculé les trajectoires rectilignes relatives de quelques couples supplémentaires, intéressants de ce point de vue (Dommanget, 1969, 1974).

Le tableau I contient les dix-huit couples ainsi disponibles et donne, en plus des valeurs des mouvements propres relatifs de leurs composantes en ascension droite et en déclinaison, déduits des trajectoires, leurs écarts avec les valeurs correspondantes déduites des trois catalogues cités.

D'après les moyennes quadratiques de ces écarts, on constate que les valeurs tirées du EBL concordent mieux avec celles calculées par les trajectoires, que les valeurs provenant des deux autres catalogues et cela, tant en ascension droite, qu'en déclinaison, comme l'illustrent les graphiques de la figure 2 . 
230 J. DOMMANGET
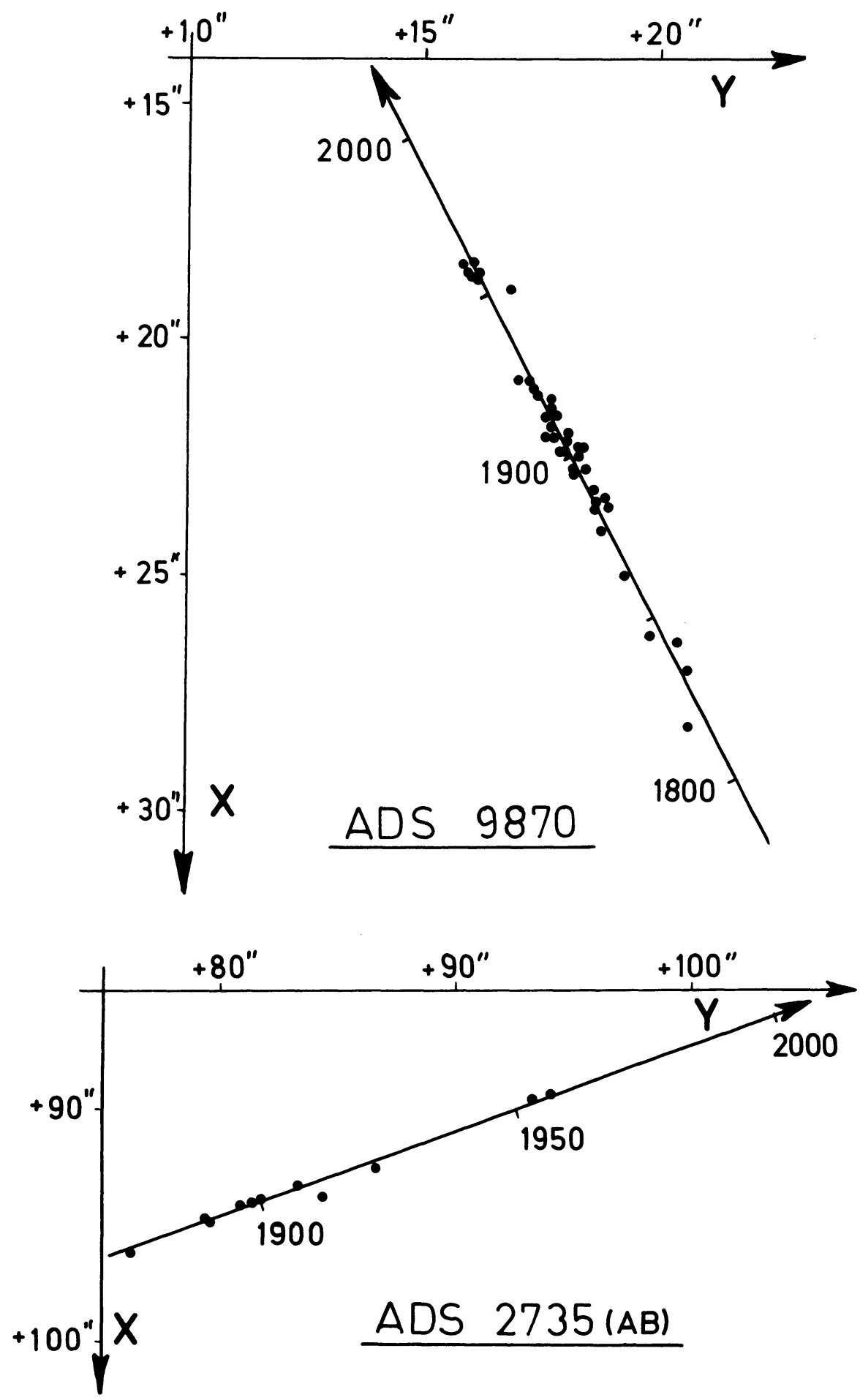

Fig. 1a, b. Exemples de représentations d'observations visuelles de couples stellaires, par des trajectoires relatives rectilignes. 
TABLEAU I

\begin{tabular}{|c|c|c|c|c|c|c|c|c|}
\hline \multirow{2}{*}{$\begin{array}{l}\text { Etoile } \\
\text { No. Cat. Index }\end{array}$} & \multicolumn{2}{|c|}{ Traj. rect. } & \multicolumn{2}{|l|}{ EBL } & \multicolumn{2}{|l|}{ Yale } & \multicolumn{2}{|l|}{ SAO } \\
\hline & $\alpha$ & $\delta$ & $\alpha$ & $\delta$ & $\alpha$ & $\delta$ & $\alpha$ & $\delta$ \\
\hline 0410 N 3024 & $-0,021$ & $+0,036$ & $+0,012$ & $+0,005$ & $+0,020$ & $+0,014$ & $-0,002$ & $-0,002$ \\
\hline $0543 \mathrm{~N} 0015(A B)$ & $+0,026$ & $+0,102$ & $-0,001$ & $+0,016$ & $+0,006$ & $-0,005$ & $+0,021$ & $-0,006$ \\
\hline $01437 \mathrm{~S} 0155(A B)$ & $-0,006$ & $-0,041$ & $+0,001$ & $-0,001$ & $+0,024$ & $-0,023$ & $+0,024$ & $+0,027$ \\
\hline 02159 N 2310 & $+0,048$ & 0,000 & $+0,003$ & 0,000 & $+0,012$ & $+0,001$ & $+0,007$ & $-0,003$ \\
\hline 3361 S 1256 & $-0,094$ & $-0,038$ & $+0,002$ & $+0,005$ & $+0,040$ & $+0,012$ & $+0,014$ & $+0,022$ \\
\hline $385 \mathrm{~N} 2735(A B)$ & $+0,218$ & $-0,081$ & $-0,008$ & $-0,027$ & $-0,033$ & $+0,013$ & $-0,025$ & $+0,028$ \\
\hline $3594 \mathrm{~N} 2144(A B)$ & $-0,165$ & $+0,068$ & $+0,003$ & $+0,008$ & $-0,008$ & $+0,003$ & $-0,008$ & $+0,014$ \\
\hline 42 N 2707 & $+0,007$ & $+0,087$ & $-0,001$ & $+0,005$ & $+0,025$ & $-0,022$ & $+0,001$ & $-0,008$ \\
\hline 05186 N 1716 & $-0,230$ & $-0,017$ & $+0,011$ & $+0,001$ & $+0,022$ & $+0,013$ & $+0,003$ & $+0,004$ \\
\hline $2218 \mathrm{~N} 2051(A B)$ & $+0,028$ & $+0,034$ & $+0,004$ & $-0,001$ & $+0,002$ & 0,000 & 0,000 & $+0,007$ \\
\hline $\mathrm{N} 0229(A B)$ & $+0,173$ & -0 , & $-0,010$ & +0 & $-0,028$ & $+0,003$ & $-0,027$ & $-0,001$ \\
\hline $09029 \mathrm{~N} 2702(A B)$ & $+0,115$ & $+0,324$ & $+0,012$ & $-0,002$ & $+0,015$ & $+0,009$ & $+0,034$ & $+0,004$ \\
\hline 09094 N $2348(A C)$ & $+0,138$ & $+0,088$ & $+0,006$ & $-0,029$ & $+0,001$ & $+0,001$ & $-0,012$ & $-0,021$ \\
\hline 488 N 0525 & $-0,020$ & $-0,030$ & $+0,003$ & $+0,002$ & $+0,004$ & $-0,016$ & $-0,011$ & $-0,010$ \\
\hline $13097 \mathrm{~S} 1050(A B)$ & $+0,221$ & $+0,311$ & $-0,012$ & $+0,009$ & $-0,037$ & $+0,020$ & $-0,014$ & $+0,003$ \\
\hline $14516 \mathrm{~S} 2058(A B)$ & $-0,058$ & $+0,082$ & $-0,032$ & $+0,008$ & $+0,035$ & $-0,020$ & $-0,011$ & $-0,010$ \\
\hline $19202 \mathrm{~N} 1143(A B)$ & $-0,679$ & $-0,707$ & $-0,012$ & $-0,022$ & $-0,002$ & $-0,024$ & $-0,014$ & $-0,032$ \\
\hline $20264 \mathrm{~N} 1055(A-B C)$ & $-0,019$ & $-0,005$ & $-0,003$ & $-0,005$ & $+0,009$ & $-0,012$ & $+0,005$ & $-0,003$ \\
\hline & & & & & & & & 16 \\
\hline
\end{tabular}
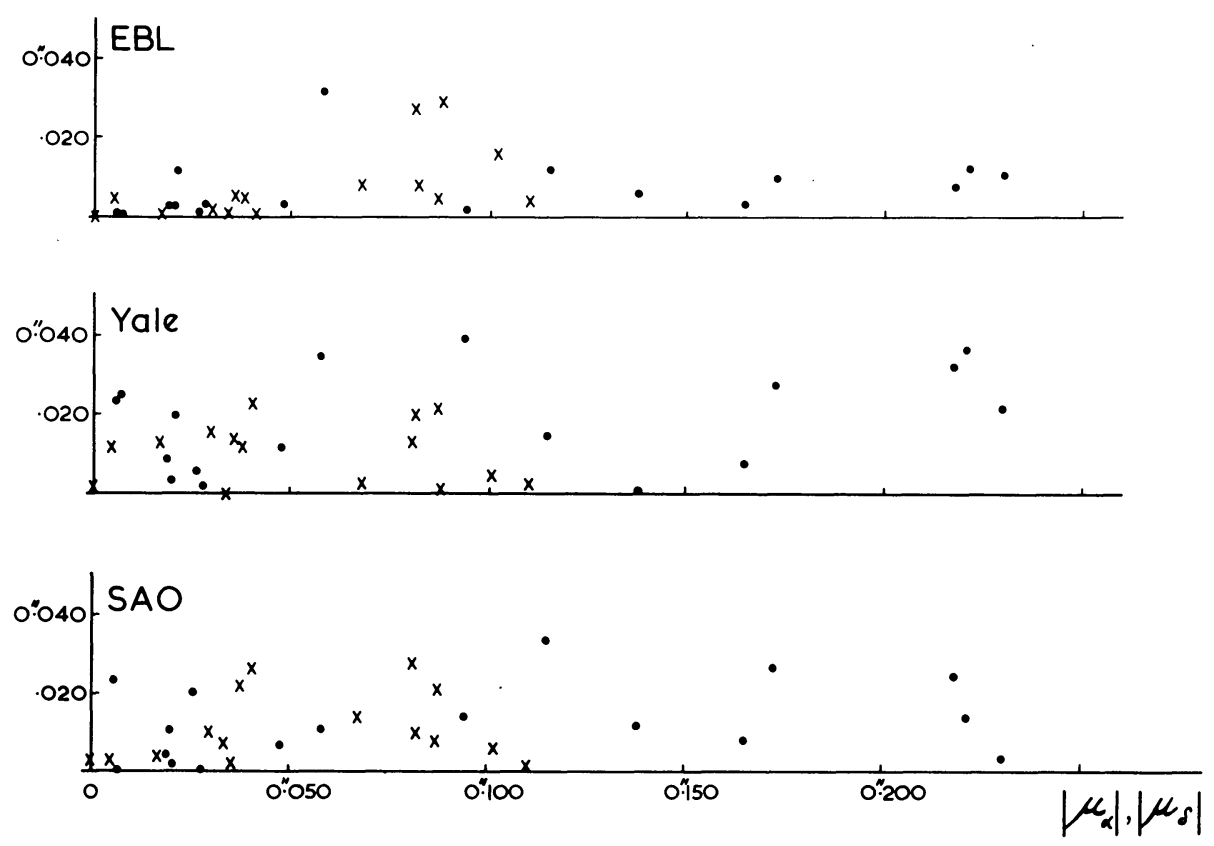

Fig. 2. Représentation graphique, pour les 18 couples stellaires considérés ici, des écarts entre les mouvements propres relatifs déduits des catalogues EBL, de Yale et du Smithsonian Astrophysical Observatory et ceux déduits des trajectoires rectilignes calculées à partir des observations visuelles. Les signes de ces écarts ainsi que ceux des valeurs de $\mu_{x}$ et de $\mu_{\delta}$ ont été ignorés. Les points correspondent aux écarts en $\alpha$, les croix aux écarts en $\delta$. 
Remarquons que tous les couples retenus présentent une distance de séparation comprise entre $15^{\prime \prime}$ et $100^{\prime \prime}$ environ, ce qui permet d'éviter d'une part les effets dûs à la proximité des images photographiques stellaires et, d'autre part, les trop grandes imprécisions entachant les mesures visuelles des couples très écartés. Par ailleurs, les observations couvrent, dans chaque cas, au moins des intervalles de temps de près d'un siècle.

Il n'empêche qu'avant de pouvoir tirer une conclusion certaine d'une telle comparaison, il y aurait lieu d'entreprendre le calcul des trajectoires relatives d'un plus grand nombre de couples pour lesquels les mouvements propres des composantes sont donnés dans les catalogues à comparer.

Aussi, des trajectoires rectilignes sont régulièrement calculées à l'Observatoire Royal de Belgique, dans ce but.

Pour terminer, nous insisterons sur le fait que la prétention de cette note se limite à montrer les possibilités de la méthode envisagée pour évaluer la qualité relative des mouvements propres donnés dans les catalogues d'étoiles.

\section{Bibliographie}

Dommanget, J.: 1969, Bull. Astron. Obs. Roy. Belgique 6, 343.

Dommanget, J. : 1974, Bull. Astron. Obs. Roy. Belgique 8, sous presse.

Dommanget, J. et Nys, O.: 1964, Ann. Obs. Roy. Belgique, 3ème Sér. 9 (6), 309. 